\title{
Resolução de Labirintos e Tarefa Agir/Não Agir na Avaliação Atentiva
}

\author{
Solving Mazes and Go/NoGo Task in Attentive Assessment
}

\author{
Joaquim Carlos Rossini*, Lorena Barbosa Cunha Macedo \& Fabiana Pires Teobaldo \\ Universidade Federal de Uberlândia, Uberlândia, $M G$, Brasil
}

\begin{abstract}
Resumo
Os processos atentivos são fundamentais para o controle do comportamento e para o planejamento da ação. Este estudo investigou uma possível relação no desempenho de participantes $(n=20)$ sem queixas clínicas na resolução de labirintos e na execução de um teste computadorizado de desempenho contínuo (Agir/Não Agir). O desempenho dos participantes foi analisado em termos de possíveis relações significativas entre o tempo médio de resolução dos labirintos e o tempo médio na execução da tarefa Agir/Não Agir, bem como uma possível relação entre os tipos de erros cometidos nas duas tarefas. Os resultados sugerem uma relação significativa entre o tempo de execução das duas tarefas e uma correlação entre a frequência de toques nos limites dos labirintos e a quantidade de erros de comissão cometidos em uma tarefa Agir/Não Agir. Esses resultados são discutidos em termos de possíveis marcadores comportamentais de ações impulsivas.

Palavras-chave: Labirintos, tarefa agir/não agir, atenção, impulsividade.
\end{abstract}

\begin{abstract}
Attentive processes are critical to behavior control and action planning. The present study investigated a possible relationship in the performance of participants $(n=20)$ without clinical complaints in solving mazes and in a computerized continuous performance test (Go/NoGo). The performance of participants was analyzed in terms of possible significant relationships between average time in the resolution of mazes and average time in implementing the Go/NoGo task, as well as a possible relationship between types of errors in both tasks. The results suggest a significant relationship between the runtime of both tasks and correlation between the frequency of touches at the limits of mazes and the amount of commission errors committed in $\mathrm{Go} / \mathrm{NoGo}$ task. These results are discussed in terms of possible behavioral markers of impulsive actions.
\end{abstract}

Keywords: Mazes, go/nogo task, attention, impulsivity.

Os processos cognitivos relacionados à atenção são responsáveis por possibilitar a ampliação do planejamento e a análise de estratégias mais apropriadas para a resolução de tarefas, permitindo a redução da ocorrência de comportamentos não planejados. De maneira geral, os processos atentivos são investigados em termos de quatro processos componentes: a capacidade de foco da atenção, a atenção seletiva, os processos de respostas e controle executivo, e atenção constante (Cohen, Salloway, \& Zawacki, 2006). No que concerne aos processos de resposta e controle executivo, especialmente relacionados aos aspectos da autorregulação do comportamento, a capacidade de inibir informações e ações inapropriadas desempenha um papel fundamental para a capacidade de manter o curso de uma ação planejada, evitando assim, atos inadequados ou a não execução de ações adaptativas.
As ações impulsivas são caracterizadas como o ato de decidir sobre determinadas questões, sem levar em consideração as consequências futuras, ou como rápidas respostas motoras, na maioria das vezes não planejadas ou fora de contexto, acompanhadas de uma dificuldade de tolerar frustrações e adiamentos de gratificações, o que frequentemente resulta em ações menos adequadas em médio e longo prazo (Araújo, Malloy-Dinniz, \& Rocha, 2009). Por sua vez, os comportamentos desatentos são caracterizados como divagações em tarefas, falta de persistência, dificuldade de manter o foco e desorganização (American Psychiatric Association, 2014). Esses dois aspectos são sistematicamente investigados por meio de tarefas comportamentais capazes de mobilizar os processos atentivos e mnemônicos envolvidos tanto na inibição de ações inadequadas quanto na manutenção eficiente de um comportamento adaptativo.

No que diz respeito à investigação dos processos cognitivos motores envolvidos no planejamento da ação em resposta a um estímulo visuoespacial, uma tarefa bastante difundida é a resolução de labirintos. Esta tarefa exige a 
mobilização de recursos executivos relacionados à seleção, integração, inibição e programação motora do comportamento, bem como de recursos da memória operacional e de longo prazo, fundamentais para a expressão de uma série de aspectos mais amplos da cognição humana, como o planejamento da ação e a resolução de problemas. Assim, os labirintos são meios legítimos na avaliação das funções executivas associadas ao planejamento e controle de respostas motoras permitindo uma avaliação dimensional desses construtos, uma vez que o grau de complexidade imposta pela tarefa está diretamente relacionada à mobilização de recursos cognitivos para a sua correta resolução. Sob o ponto de vista neuropsicológico, Ott et al. (2008) e Ott et al. (2003), utilizaram testes de labirintos computadorizados na avaliação de grupos de idosos saudáveis ou diagnosticados com a doença de Alzheimer no contexto do trânsito. Os participantes realizaram primeiramente um teste de condução de veículo na presença de um avaliador, o qual registrava o desempenho na tarefa. Em um momento posterior, os mesmos participantes foram submetidos a um teste computadorizado de labirintos. O resultado dessa investigação evidenciou uma relação consistente entre a habilidade na resolução do teste de labirintos e o desempenho na avaliação prática de motoristas idosos. Krishnasamy e Unsworth (2011) utilizaram os labirintos presentes no "The Drive Home Maze Test" e concluíram que a probabilidade de erros cometidos em exames práticos de motoristas apresentou uma relação significativa com o tempo de resolução de labirintos, resultados estes que corroboram os achados de Ott et al. (2008). Recentemente, Ott et al. (2013) investigaram a sensibilidade e a especificidade de testes para a avaliação de indivíduos idosos no que concerne as suas habilidades perceptivas, cognitivas e motoras para a condução de veículos. Esta bateria de testes incluía o protocolo de avaliação de habilidade relacionadas à condução veicular (Assessment of Driving-Related Skills [ADReS]), tarefas de resolução de labirintos (tradicional, com o uso de lápis e papel, ou computadorizada, Snellgrove Maze Task), além da realização do Teste das Trilhas (Parte A e B). O estudo foi realizado com a participação de 122 idosos com idade entre 60 e 90 anos, com e sem prejuízos cognitivos. Os resultados gerais apontam uma melhora no poder preditivo da bateria de avaliação em relação às habilidades cognitivas necessárias para a condução veicular com a inclusão da tarefa de resolução de labirintos no protocolo de avaliação.

Na mesma linha investigativa, Barco, Wallendorf, Snellgrove, Ott e Carr (2014) apresentaram novos resultados que apoiam a utilização da tarefa de resolução de labirintos na avaliação cognitiva das funções executivas no contexto do transito veicular. Nesse estudo, da mesma maneira que Ott et al., 2013, os autores observaram o valor preditivo de uma ampla bateria de testes cognitivos, visuais e motores no desempenho de sujeitos vítimas de acidente vascular cerebral (AVC) nas habilidades requeridas para a condução de veículos. O resultado dessa investigação também sugere que o melhor protocolo de avaliação nesses casos inclui tarefas de resolução de labirintos de Snellgrove e o teste de trilhas (Parte B).

Em âmbito nacional, Souza, França e Campos (2006) investigaram a tarefa de resolução de labirintos no contexto da Fisioterapia. Os autores investigaram a curva de desempenho na aquisição e persistência da habilidade motora de resolução de labirintos. Esse estudo foi realizado com a participação de 70 sujeitos saudáveis do sexo masculino. Os participantes foram divididos em dois grupos compostos por 35 participantes. Aos participantes foi solicitada a realização de um labirinto apresentado 30 vezes, solucionados um de cada vez após um sinal sonoro. O labirinto apresentado a um dos grupos apresentava pistas visuais das possíveis rotas de solução. Os participantes realizavam novamente a resolução do labirinto após um intervalo de 15 minutos e a realização de um teste de memória com dígitos. Foi registrado o tempo para completar a tarefa e o número de tentativas para a estabilização do desempenho. Os resultados indicaram que os dois tipos de labirintos, com e sem a presença de pistas visuais, resultaram em uma curva de desempenho com grande variação no desempenho nas provas iniciais e pouca variação nas provas finais da prática. O desempenho manteve-se estável após a tarefa de memorização de dígitos e o intervalo de retenção em ambos os tipos de labirintos. Baseados nesses resultados os autores sugerem que a tarefa de resolução de labirintos, com e sem pistas, pode ser um instrumento válido na análise do processo de aquisição de uma habilidade motora e útil na composição de protocolos de reabilitação. Em outro estudo, Silva (2008) utilizou o teste de labirintos (realizados no papel) para a avaliação cognitiva em indivíduos idosos que apresentavam ou não comprometimentos neuropsicológicos. Os resultados desta investigação mostraram que idosos diagnosticados com a doença de Alzheimer apresentavam um aumento significativo no tempo de resolução dos labirintos, quando comparados a idosos saudáveis. No que concerne a utilização do teste de labirintos na investigação das funções executivas em diferentes faixas etárias, Souza, Oliveira e Campos (2013) apresentam resultados que indicam a viabilidade do uso dessa tarefa na avaliação dos processos motores e mnemônicos em adultos jovens e idosos. Esse estudo foi realizado com a participação de 80 sujeitos saudáveis, de ambos os sexos, divididos em dois grupos etários compostos por 40 sujeitos jovens e 40 sujeitos idosos. Assim como no procedimento adotado por Souza et al. (2006), os participantes de ambos os grupos foram solicitados a resolver sequencialmente 30 vezes o mesmo labirinto. O labirinto utilizado na tarefa apresentava apenas uma entrada e uma saída, mas contava com seis possíveis rotas de resolução, sendo que apenas uma rota de resolução era a mais eficiente. Após um intervalo de 15 minutos, os participantes foram testados novamente com o mesmo labirinto. Os resultados obtidos indicaram que o tempo de execução da tarefa foi significativamente menor para os participantes jovens quando comparados ao tempo de execução apresentados pelos idosos. Os resultados também apontaram uma diferença significativa entre o tempo 
de execução do último labirinto da série de 30 resoluções e o tempo de execução do labirinto teste após o intervalo de 15 minutos para o grupo de idosos, o que pode sugerir um comprometimento na manutenção do desempenho adquirido em função do aumento da idade.

Desta forma, um número expressivo de estudos acerca da tarefa de resolução de labirintos ressalta a influência significativa de aspectos relacionados aos processos cognitivos fundamentais como a seleção e manutenção do foco atentivo e a memória operacional, bem como a fatores mais amplos como a capacidade de planejamento, resolução de problemas e programação e execução de respostas motoras. Sob o ponto de vista prático, como sugerido por Silva (2008), esta tarefa apresenta uma grande praticidade em sua aplicação, podendo ser resolvidos com materiais simples (papel e lápis) ou mais sofisticados (versões computadorizadas), o que permitiria sua utilização em contextos múltiplos, como em diversas atividades da área da saúde, além de não requererem grandes períodos de tempo para a sua execução. Uma questão importante, no entanto, é o estabelecimento de tarefas, que realizadas juntamente ao teste de labirintos, possam ampliar a compreensão dos processos atentivos envolvidos, tornando possível a avaliação de fatores mais amplos, como a desatenção ou a impulsividade.

Neste sentido, uma tarefa bastante promissora na investigação dos processos envolvidos nos comportamentos impulsivos e de autorregulação da emissão de respostas motoras, e que tradicionalmente não é realizada juntamente a tarefa de resolução de labirintos, é a avaliação nos Testes de Desempenho Contínuo (TDC), também conhecidos como tarefas Agir/Não Agir (Go/NoGo Task; Bezdjian, Baker, Lozano, \& Raine, 2009; Logan, 1994; Miranda, Sinnes, Pompeia, \& Bueno, 2009; Riccio, Reynolds, \& Lowe, 2001; Steele et al., 2013). Nessa tarefa os participantes são instruídos a responder, pressionando o mais rápido possível uma tecla do computador, na presença de um estímulo alvo, (eg., letras = estímulos agir) e não executar nenhuma resposta na presença de um estímulo não agir (eg., números = estímulos não agir). Esta tarefa permite a observação de três aspectos importantes na avaliação do comportamento: a omissão em efetuar uma resposta esperada, a execução de uma resposta indevida (comissão), e o tempo despendido pelo participante para efetuar uma resposta. A frequência de erros de omissão pode ser relacionada à ação desatenta na execução da tarefa. Por sua vez, a frequência de erros de comissão é frequentemente relacionada à impulsividade e a falha na inibição de uma resposta prepotente. O tempo de reação dos participantes está relacionado a velocidade do processamento da informação (tempo de reação motor; Bari \& Robbins, 2013; Bezdjian et al., 2009; Verbruggen \& Logan, 2008).

Em um estudo que utilizou a técnica de neuroimagem por ressonância magnética funcional Goya-Maldonado et al. (2010) investigaram uma possível relação entre respostas motoras impulsivas e as áreas cerebrais ativadas durante o processo de inibição da resposta em uma tarefa
Agir/Não Agir. Os resultados desse estudo sugerem uma correlação positiva entre a impulsividade motora e a ativação de áreas do córtex pré-frontal ventrolateral durante inibições comportamentais bem sucedidas. Esse resultado corrobora a interpretação que importantes áreas do córtex pré-frontal estão envolvidas tanto na modulação de respostas inibitórias (Bari \& Robbins, 2013) quanto nos processos de planejamento e autorregulação do comportamento em tarefas de resolução de labirintos visuoespaciais (Kirsch et al., 2006).

O presente estudo investigou uma possível relação no desempenho de participantes, sem queixas clínicas, na resolução de uma série de labirintos e na execução de um teste computadorizado de desempenho contínuo (Agir/Não Agir). O desempenho dos participantes foi analisado em termos de possíveis relações significativas entre o tempo médio de resolução dos labirintos e o tempo médio na execução da tarefa Agir/Não Agir, bem como uma possível relação entre os tipos de erros cometidos nas duas tarefas.

\section{Método}

\section{Participantes}

A pesquisa contou com a participação voluntária de 20 sujeitos (11 do sexo feminino), sem queixas clínicas, com idades entre 19 e 28 anos $(M=24,6, D P=2)$. Quanto à escolaridade, $33,33 \%$ dos sujeitos cursavam o ensino superior e $66,6 \%$ possuíam ensino superior completo. A amostra foi selecionada por conveniência e os critérios de exclusão para esta participação foram: problemas de visão não corrigidos, problemas neurológicos diagnosticados, transtornos do sono, déficits motores, uso de drogas ou álcool, uso de medicamentos neurológicos ou psiquiátricos, assim como histórico clinico de epilepsia e traumatismo crânio-encefálico e transtornos mentais sem o uso de medicação. Estas informações foram obtidas por meio de dois questionários, um sociodemográfico e outro biomédico, respondidos pelos participantes antes da execução das tarefas. Com o intuito de identificar possíveis dificuldades na habilidade visuoespacial, cada participante foi solicitado a responder o teste de Matrizes Progressivas de Raven (Forma Geral; Raven, 2000). O desempenho nesse teste não foi utilizado como critério eliminatório e todos os participantes obtiveram escores entre médio e médio superior nesta avaliação.

\section{Instrumentos e Procedimentos}

As tarefas foram realizadas individualmente em apenas uma sessão de aproximadamente 50 minutos. Inicialmente os participantes foram informados sobre o propósito da investigação e firmaram o Termo de Consentimento Livre e Esclarecido (TCLE). O protocolo de avaliação utilizado foi aprovado pelo Comitê de Ética em Pesquisa (CAAE18542313.2.0000.5152). No início da sessão os participantes responderam a dois questionários, um apresentando questões sociodemográficas e outro apresentando questões biomédicas gerais. A tarefa Agir/Não Agir e a resolução 
Rossini, J. C., Macedo, L. B. C. \& Teobaldo, F. P. (2015). Resolução de Labirintos e Tarefa Agir/Não Agir na Avaliação Atentiva.

dos Labirintos foram realizadas em ordem aleatória entre os participantes.

Tarefa Agir/Não Agir. Os estímulos da tarefa Agir/ Não Agir foram apresentados em um computador, tela 14 polegadas. O software E-Prime 2.0 (Schneider \& Zuccoloto, 2007) foi utilizado na programação e apresentação dos estímulos. O mesmo aplicativo registrou os tempos de reação (TRs) e os erros cometidos durante a tarefa. Os estímulos visuais foram compostos por números de $1 \mathrm{a}$ 9, apresentados no centro da tela do computador. Cada prova era iniciada com a apresentação de um ponto de fixação $(+)$, durante $1000 \mathrm{~ms}$, seguido pela apresentação pseudoaleatória de números de $1 \mathrm{a} 9$. $\mathrm{O}$ mesmo número nunca era apresentado imediatamente em sequência. Cada número foi apresentado por $166 \mathrm{~ms}$. Os números eram apresentados na cor preta $\left(0,02 \mathrm{~cd} / \mathrm{m}^{2}\right)$ sobre o fundo branco da tela do computador $\left(100 \mathrm{~cd} / \mathrm{m}^{2}\right)$. A tarefa do participante era pressionar a barra de espaço do teclado do computador, o mais rápido possível, após a apresentação dos números: 1, 2, 4, 5, 6, 7, 8, e 9 (estímulos agir). O participante era instruído a não pressionar a barra de espaço na presença do número 3 (estímulo não agir). Após a apresentação do número, o caractere (\#) na cor preta era apresentado como uma máscara visual até a resposta do participante ou por um intervalo de $1000 \mathrm{~ms}$. Em seguida, dependendo da resposta ou não do participante o caractere (\#) era apresentado na cor azul $\left(5 \mathrm{~cd} / \mathrm{m}^{2}\right)$, caso o participante pressionasse a tecla corretamente, ou (\#) na cor vermelha $\left(5 \mathrm{~cd} / \mathrm{m}^{2}\right)$, caso o participante pressionasse a tecla incorretamente. O caractere (@) na cor azul era apresentado quando o participante não pressionava a tecla após a apresentação do número "3" (estímulo Não Agir). Os caracteres de feedback eram apresentados por $500 \mathrm{~ms}$ e imediatamente após a sua apresentação uma nova prova era iniciada. A Figura 1A exemplifica a sequência de eventos em uma prova. Cada participante realizou 20 provas treino e 200 provas experimentais (150 provas Agir e 50 provas Não Agir).

Teste de Labirintos. Os labirintos foram criados para a presente investigação sendo compostos por 5 matrizes com mesmo nível de dificuldade. Cada labirinto foi impresso centralizado em uma folha A4 com dimensões de 12x12 centímetros. A complexidade dos labirintos foi definida em função do número de escolhas até o centro da matriz.

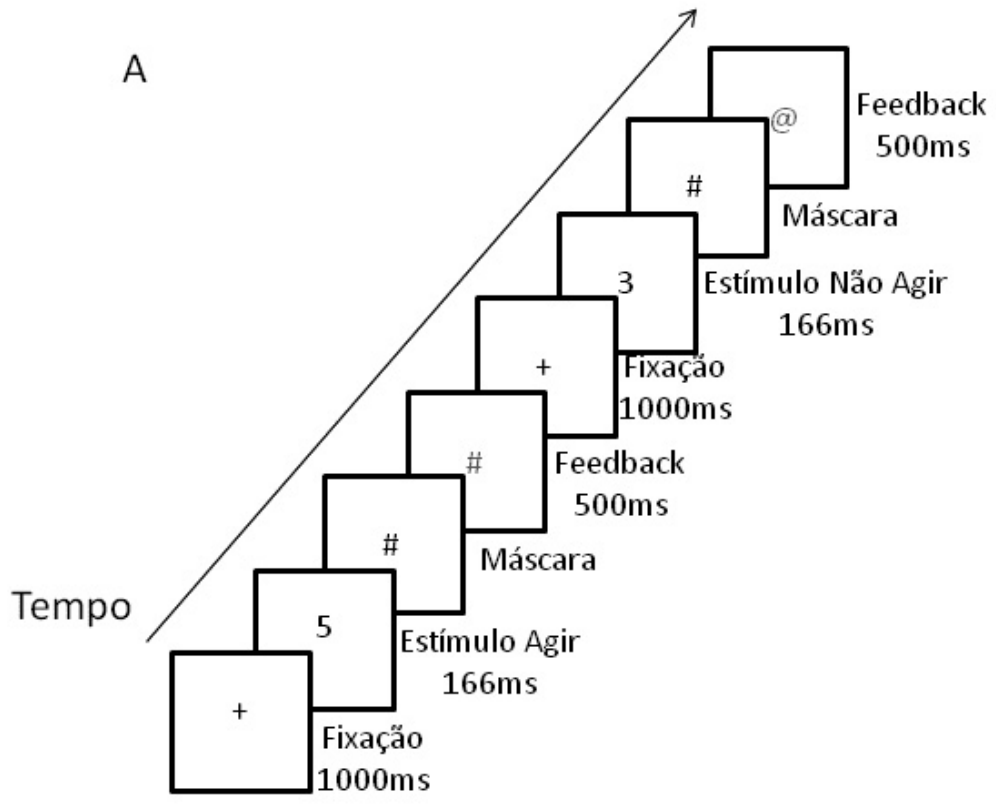

B 
A Figura 1B exemplifica os labirintos desenvolvidos para esta tarefa. A tarefa do participante era traçar, o mais rápido possível, um caminho da entrada do labirinto até seu centro, sem retirar o lápis da folha de papel. Durante a execução dessa prova, o tempo de resolução de cada labirinto foi registrado por meio do software E-Prime 2.0 (Schneider \& Zuccoloto, 2007). Os participantes foram orientados a encontrar o caminho adequado de chegada ao centro do labirinto, o mais rápido possível, traçando uma trajetória contínua. Caso o sujeito optasse por alterar a trajetória do traçado, ele era orientado a retornar com o traço até o ponto do traçado que julgasse adequado para a nova estratégia de resolução. $O$ registro do tempo de execução era iniciado no momento em que o labirinto era apresentado ao participante e encerrado no momento que o participante finalizava a tarefa (finalizando o traçado no centro do labirinto).

\section{Resultados}

O desempenho na tarefa Agir/Não Agir foi analisada em função das seguintes variáveis: tempo de reação (TR), erros de comissão (EC), e erros de omissão (EO). Foram realizadas análises da variância (ANOVA) entre os fatores independentes sexo e idade e as variáveis dependentes avaliadas. Tal análise não confirmou um efeito significativo dos fatores independentes no desempenho da tarefa Agir/ Não Agir. O TR médio dos participantes na tarefa foi igual a $244 \mathrm{~ms}(D P=75)$, a porcentagem de $\mathrm{EC}$ foi igual a $27,8 \%$ e a porcentagem de EO foi igual a $10,3 \%$.

Uma análise da regressão linear entre os tipos de erros cometidos confirmou uma relação inversa entre os mesmos,
$R_{\text {ajustado }}^{2}=0,93, F(1,18)=269,75, p<0,001, \beta=-0,97$. Houve uma relação inversa entre o TR e a ocorrência de EC, ou seja, quanto menor o tempo médio de resposta apresentado pelo participante maior a probabilidade de realizar respostas inapropriadas, $R_{\text {ajustado }}^{2}=0,22, F(1,18)=$ $6,37, p=0,02, \beta=-0,51$. A mesma análise evidenciou uma relação significativa e direta entre o TR e a ocorrência de omissões, $R_{\text {ajustado }}^{2}=0,24, F(1,18)=7,23, p=0,01, \beta=0,53$.

$\mathrm{O}$ desempenho dos participantes na resolução dos labirintos foi analisado em função do desempenho médio dos participantes na tarefa. Foram realizadas análises da variância (ANOVA) entre os fatores sexo e idade e as variáveis dependentes avaliadas. Tal análise confirmou um efeito significativo do fator sexo no tempo de resolução dos labirintos confirmando que, na amostra investigada, os participantes do sexo masculino foram, em média mais rápidos na execução da tarefa, $F(1,18)=5,19, p=0,03$, $\eta_{\mathrm{p}}^{2}=0,22\left(\mathrm{TR}_{\text {masculino }}=5 \mathrm{~s}, D P=2 ; \mathrm{TR}_{\text {feminino }}=7,4 \mathrm{~s}, D P=\right.$ $2,5)$. Esse resultado pode sugerir uma especificidade em relação ao fator sexo na resolução de tarefas visuoespaciais mais abrangentes como a resolução de labirintos.

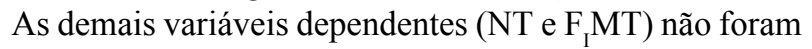
influenciadas significativamente por este fator $(p>0,05)$. A mesma análise não confirmou uma influência significativa do fator idade sobre nenhuma das variáveis dependentes investigadas $(p>0,05)$. Uma análise da regressão linear entre as variáveis $\mathrm{NT}$ e $\mathrm{F}_{1} \mathrm{MT}$ evidenciou uma associação positiva entre ambas, $R_{\text {ajustado }}^{2}=0,17, F(1,18)=5,13, p=$ $0,03, \beta=0,47$.

A correlação produto-momento de Pearson do desempenho dos participantes foi analisada, como sumariado na Tabela 1.

Tabela 1

Sumário das Inter Correlações entre o Desempenho dos Participantes na Tarefa Agir/Não Agir e na Tarefa de Resolução de Labirintos

\begin{tabular}{cccccccc}
\hline VD & 1 & 2 & 3 & 4 & 5 & 6 \\
\hline 1. & TR & - & & & & \\
2. & EC & $-0,51^{*}$ & - & & & \\
3. & EO & $0,53^{*}$ & $-0,96^{*}$ & - & & \\
4. & TRL & $0,46^{*}$ & $-0,15$ & 0,18 & - & \\
5. & NT & $-0,51^{*}$ & $0,59^{*}$ & $-0,64^{*}$ & $-0,13$ & - & \\
6. & FIMT & $-0,23$ & 0,19 & $-0,16$ & 0,20 & $0,47^{*}$ & - \\
\hline
\end{tabular}

Nota. Tarefa Agir/Não Agir: Tempo de Reação (TR), Erros de Comissão (EC), Erros de Omissão (EO). Tarefa de resolução de Labirintos: Tempo de resolução dos labirintos (TRL), número de toques (NT) e número de finalizações incorretas e mudanças de trajetória $\left(\mathrm{F}_{\mathrm{I}} \mathrm{MT}\right)$.

${ }^{*} p<0,05$.

Houve uma correlação positiva e significativa entre o tempo médio de resposta motora na tarefa Agir/Não Agir e o tempo médio de resolução dos labirintos, $r=0,46$, o que sugere uma relação no tempo de execução nas duas tarefas, ou seja, participantes mais lentos na tarefa de resolução de labirinto tendem a serem mais lentos na tarefa Agir/Não 
Agir. O TR Agir/Não Agir apresentou uma correlação inversa em relação ao número de erros de comissão Agir/ Não Agir, $r=-0,51$, apontando que participantes mais rápidos nesta tarefa tendem a cometer mais erros desse tipo. Esta análise também confirmou uma relação direta entre o TR dos participantes na tarefa Agir/Não Agir e o número de erros de omissão cometidos, $r=0,53$, o que sugere que participantes mais lentos tendem a cometer mais erros desse tipo. Foi observada uma relação inversa entre os erros de comissão e omissão, $r=-0,96$, sugerindo que os participantes que incorreram em um tipo de erro, não o fizeram no outro tipo de erro. É interessante notar que o tempo de reação na tarefa Agir/Não Agir e o tempo de resolução dos labirintos apresentam uma correlação inversa ao número de toques, $r=-0,51$ e $r=-0,13$ (ns), respectivamente, sugerindo que sujeitos mais rápidos tendem a apresentar uma frequência maior de toques nas paredes dos labirintos.

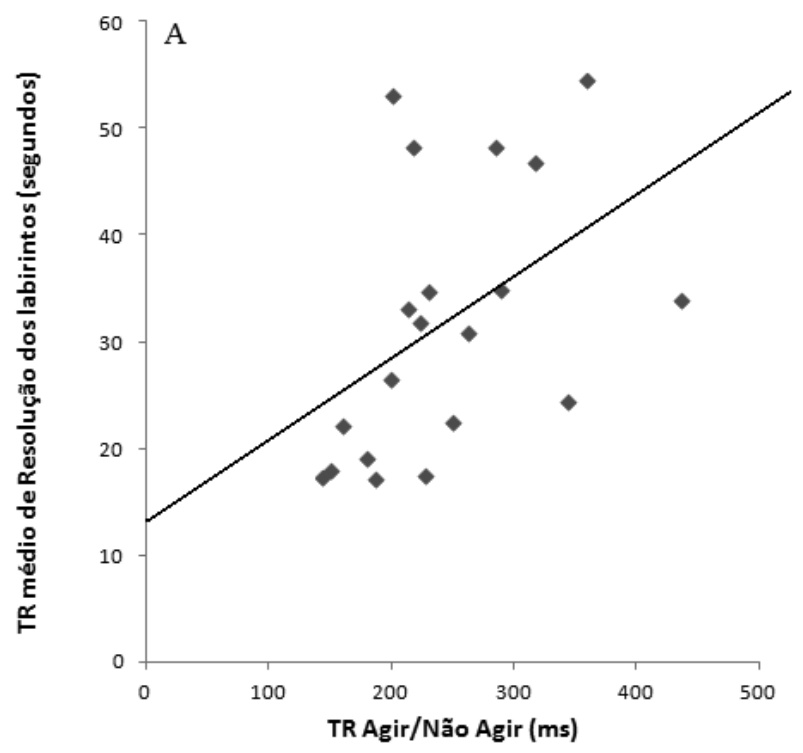

O número de comissões no teste Agir/Não Agir mostra uma correlação inversa ao número de omissões cometidos no mesmo teste. Mais importante, a ocorrência de erros de comissão no teste Agir/Não Agir apresenta uma correlação direta com o número de toques durante a resolução dos labirintos, $r=0,59$. Por outro lado, há uma relação inversa entre o número de omissões no teste Agir/Não Agir e o número de toques durante a resolução dos labirintos, $r=$ $-0,64$. Houve uma correlação positiva entre a ocorrência de becos e mudança de trajetória, $r=0,53$. Uma análise da regressão linear entre os tempos de execução das duas tarefas confirmou uma relação significativa, $R_{\text {ajustado }}^{2}=$ $0,17, F(1,18)=4,89, p<0,05, \beta=0,46$, como mostra a Figura $2 \mathrm{~A}$. O número de erros de comissão foi analisado em função do número de toques nas paredes dos labirintos confirmando uma relação significativa entre essas variáveis dependentes, $R_{\text {ajustado }}^{2}=0,32, F(1,18)=9,96, p<0,01, \beta=$ 0,60 , como mostra a Figura 2 B.

Figura 2. (A) Regressão linear do tempo de reação TR (ms) na tarefa Agir/Não Agir em função do tempo médio de resolução dos labirintos (em segundos). (B) Regressão linear do número de erros de comissão na Tarefa Agir/Não Agir em função do número médio de toques nas paredes dos labirintos.

\section{Discussão}

Uma questão central na investigação dos processos cognitivos é o estabelecimento de instrumentos de avaliação capazes de mensurar adequadamente comportamentos representativos de construtos amplos como a memória e os processos atentivos. Neste sentido, a presente pesquisa teve como objetivo investigar uma possível relação entre o desempenho na resolução de labirintos e os padrões de resposta em um teste de desempenho contínuo (Agir/Não Agir), sendo este último um teste promissor na investigação dos processos atentivos em situações clínicas (Barkley et al., 2008; Bezdjian et al., 2009; Logan, 1994; Miranda et al., 2009; Riccio et al., 2001; Steele et al., 2013). Os

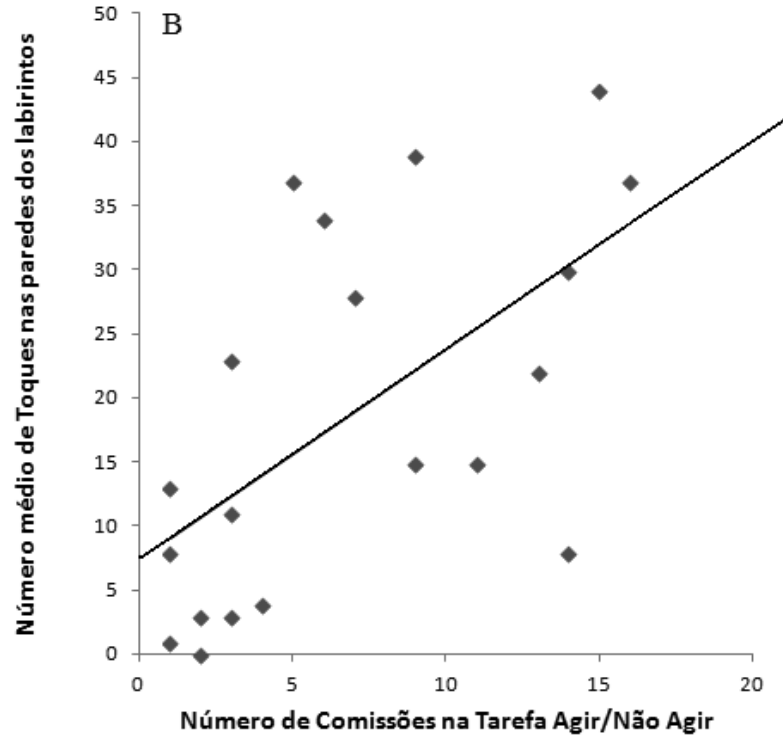


atentivos envolvidos nas tarefas propostas. Segundo Ávila-Batista e Marín Rueda (2011), a impulsividade está relacionada à tendência em efetuar respostas rápidas, sem prévia consideração acerca das possíveis consequências do comportamento, além de uma dificuldade em planejar ações efetivas, bem como em manter o foco atentivo. Nesse sentido, respostas motoras excessivamente rápidas associadas a uma quantidade elevada de erros pode sugerir uma característica impulsiva acentuada. Todavia, uma futura ampliação da presente investigação com a participação de amostras clínicas poderá evidenciar mais adequadamente essa eventual relação. No que diz respeito ao significado cognitivo dos erros cometidos, Bezdjian et al. (2009) sugerem que os erros de comissão observados na tarefa Agir/ Não Agir (agir de maneira inadequada) estão relacionados à limitação no controle do comportamento impulsivo. Os nossos resultados sugerem uma associação significativa entre o erro de comissão cometida na tarefa Agir/Não Agir e a frequência de toques nas paredes dos labirintos durante a resolução dessa tarefa. Uma vez que a relação entre a ocorrência de erros de comissão e comportamentos impulsivos é bem estabelecida, o presente resultado permite supor que a ocorrência do comportamento de toques nas paredes do labirinto também esteja relacionada às características de impulsividade durante a execução da tarefa de resolução de labirintos.

Por sua vez, os erros de omissão na tarefa Agir/Não Agir no qual o sujeito deveria apresentar uma resposta motora mas não a efetua, estão relacionados à lentificação motora e a comportamentos desatentos. Os nossos resultados evidenciaram uma relação significativa entre o tempo de execução dos participantes na realização das duas tarefas, o que sugere que a lentificação na resolução de labirintos pode representar aspectos do comportamento desatento. Esse resultado, no entanto, exige uma interpretação cautelosa, uma vez que a análise do tempo de resolução dos labirintos evidenciou uma influência significativa do fator sexo, o que pode sugerir especificidades de resolução relacionadas à característica visuoespacial desse tipo de tarefa, como sugerem outras investigações acerca das diferenças de processamento da informação em função do fator sexo (Clements-Stephens, Rimrodt, \& Cutting, 2009; Hugdahl, Thomsen, \& Ersland, 2006; Rubia, Hyde, Halari, Giampietro, \& Smith, 2010).

\section{Conclusão}

De maneira geral, os resultados evidenciam uma interessante relação entre o desempenho dos participantes na tarefa Agir/Não Agir e na tarefa de Resolução de Labirintos, sugerindo marcadores comportamentais na execução das duas tarefas que podem estar relacionados a execução de respostas impulsivas e desatentas. Apesar da limitação do presente estudo quanto ao número de participantes, a relação significativa entre o tempo de reação e a ocorrência de erros de comissão na tarefa Agir/Não Agir e o tempo de resolução de labirintos e número de toques em seus limites (paredes) sugere que a tarefa de resolução de labirintos, aliada a outras evidencias comportamentais, pode vir a ser um instrumento promissor na investigação dos processos cognitivos relacionados ao planejamento da ação comportamental.

\section{Referências}

American Psychiatric Association. (2014). Manual diagnóstico e estatístico de transtornos mentais: DSM-5. Porto Alegre, RS: Artmed.

Araújo, M. M., Malloy-Diniz, L. F., \& Rocha, F. L. (2009). Impulsividade e acidentes de trânsito. Revista de Psiquiatria Clínica, 36(2), 60-68. Recuperado em http://www.scielo.br/ pdf/rpc/v36n2/04.pdf

Ávila-Batista, A. C., \& Marín Rueda, F. J. (2011). Construção e estudos psicométricos de uma Escala de Avaliação da Impulsividade. Psico USF, 16(3), 285-295. doi:10.1590/ S1413-82712011000300005

Barco, P. P., Wallendorf, M. J., Snellgrove, C. A., Ott, B. R., \& Carr, D. B. (2014). Predicting road test performance in drivers with stroke. The American Journal of Occupational Therapy, 68(2), 221. doi:10.5014/ajot.2014.008938

Bari, A., \& Robbins, T. W. (2013). Inhibition and impulsivity: Behavioral and neural basis of response control. Progress in Neurobiology, 108, 44-79. doi:10.1177/0891988707311031

Barkley, R. A., Anastopoulos, A. D., Robin, A. L., Lovett, B. J., Smith, B. H., Cunningham, C. E., ...Hathway, W. (2008). Transtorno de déficit de atenção/hiperatividade: Manual para diagnostico e tratamento (3. ed.). Porto Alegre, RS: Artmed.

Bezdjian, S., Baker, L. A., Lozano, D. I., \& Raine, A. (2009). Assessing inattention and impulsivity in children during the Go/NoGo task. British Journal of Developmental Psychology, 27(2), 365-383. doi:10.1348/026151008X314919

Clements-Stephens, A. M., Rimrodt, S. L., \& Cutting, L. E. (2009). Developmental sex differences in basic visuospatial processing: Differences in strategy use? Neuroscience letters, 449(3), 155-160. doi:10.1016/j.neulet.2008.10.094

Cohen, R. A., Salloway, S., \& Zawacki, T. (2006). Aspectos neuropsiquiátricos dos transtornos de atenção. In S. C. Yudofsky \& R. E. Hales, Neuropsiquiatria e neurociências na prática clínica (4. ed., pp. 417-445). Porto Alegre, RS: Artmed.

Goya-Maldonado, R., Walther, S., Simon, J., Stippich, C., Weisbrod, M., \& Kaiser, S. (2010). Motor impulsivity and the ventrolateral prefrontal cortex. Psychiatry Research: Neuroimaging, 183, 89-91. doi:10.1016/j.pscychresns.2010.04.006

Hugdahl, K., Thomsen, T., \& Ersland, L. (2006). Sex differences in visuo-spatial processing: An fMRI study of mental rotation. Neuropsychologia, 44(9), 1575-1583. doi:10.1016/j. neuropsychologia.2006.01.026

Kirsch, P., Lis, S., Esslinger, C., Gruppe, H., Danos, P., Broll, J., ...Gallhofer, B. (2006). Brain activation during mental maze solving. Neuropsychobiology, 54, 51-58. doi:10.1159/000095742

Krishnasamy, C., \& Unsworth, C. A. (2011). Normative data, preliminary inter-rater reliability and predictive validity of the Drive Home Maze Test. Clinical Rehabilitation, 25(1), 88-95. doi:10.1177/0269215510382846

Logan, G. D. (1994). On the ability to inhibit thought and action - A user's guide to the stop signal paradigm. In D. Dagenbach \& T. H. Carr (Eds.), Inhibitory processes in attention, memory, and language (pp. 189-239). San Diego, CA: Academic Press. 
Miranda, M. C., Sinnes, E. G., Pompeia, S., \& Bueno, O. F. A. (2009). O K-CPT em uma amostra brasileira: Descrição do desempenho e comparação com as normas norte-americanas. Revista de Psiquiatria do Rio Grande do Sul, 31(1), 60-66. Recuperado em http://www.scielo.br/pdf/rprs/v31n1/ v31n1a11

Ott, B. R., Davis, J. D., Papandonatos, G. D., Hewitt, S., Festa, E. K., Heindel, W. C., ...Carr, D. B. (2013). Assessment of driving related skills prediction of unsafe driving in older adults in the office setting. Journal of the American Geriatrics Society, 61(7), 1164-1169. doi:10.1111/jgs.12306

Ott, B. R., Festa, E. K., Amick, M. M., Grace, J., Davis, J. D., \& Heindel, W. C. (2008). Computerized maze navigation and on-road performance by drivers with dementia. Journal of Geriatric Psychiatry and Neurology, 21(1), 18-25. doi:10.1177/0891988707311031

Ott, B. R., Heindel, W. C., Whelihan, W. M., Caron, M. D., Piatt, A. L., \& DiCarlo, M. A. (2003). Maze test performance and reported driving ability in early dementia. Journal of Geriatric Psychiatry and Neurology, 16(3), 151-155. doi:10.1177/0891988703255688

Raven, J. C. (2000). Matrizes progressivas: Escala geral (F. Campos, Trad.). Rio de Janeiro, RJ: Centro de Psicologia Aplicada. (Original publicado em 1965)

Riccio, C. A., Reynolds, C. R., \& Lowe, P. A. (2001). Clinical applications of continuous performance tests: Measuring attention and impulsive responding in children and adults. New York: John Wiley \& Sons.

Rubia, K., Hyde, Z., Halari, R., Giampietro, V., \& Smith, A. (2010). Effects of age and sex on developmental neural networks of visual-spatial attention allocation. Neuroimage, 51(2), 817-827. doi:10.1016/j.neuroimage.2010.02.058

Schneider, E., \& Zuccoloto, A. (2007). E-prime 2.0 [Computer software]: Pittsburg, PA: Psychological Software Tools.

Silva, M. H. A. F. D. (2008). Acurácia do teste de labirinto no rastreamento de déficits cognitivos em idosos (Dissertação de mestrado). Recuperado em http://repositorio.ufrn.br:8080/ jspui/bitstream/1/9232/1/MarceloHAFS DISSERT.pdf

Souza, D. E., França, F. R., \& Campos, T. F. (2006). Teste de labirinto: Instrumento de análise na aquisição de uma habilidade motora. Brazilian Journal of Physical Therapy, 10(3), 355-360. doi:10.1590/S1413-35552006000300016

Souza, D. E., Oliveira, D. C., \& Campos, T. F. (2013). Maze test: An instrument to evaluate age-related cognitive and motor changes in humans. Revista Brasileira de Ciências do Envelhecimento Humano, 10(1), 19-29. doi:10.5335/ rbceh.2013.3015

Steele, V. R., Aharoni, E., Munro, G. E., Calhoun, V. D., Nyalakanti, P., Stevens, M. C., ...Kiehl, K. A. (2013). A large scale $(N=102)$ functional neuroimaging study of response inhibition in a Go/NoGo task. Behavioural Brain Research, 256, 529-536. doi:10.1016/j.bbr.2013.06.001

Verbruggen, F., \& Logan, G. D. (2008). Automatic and controlled response inhibition: Associative learning in the go/no-go and stop-signal paradigms. Journal of Experimental Psychology: General, 137(4), 649. doi:10.1037/a0013170 